PTU-056 THE EFFECT OF HEPATITIS C INFECTION ON THE UTILITY OF ALPHA-FETOPROTEIN AS A SURVEILLANCE MARKER FOR TUMOUR RECURRENCE FOLLOWING LIVER TRANSPLANTATION FOR HEPATOCELLULAR CARCINOMA

doi:10.1136/gutjnl-2012-302514c.56

J R L Maggs, ${ }^{*}$ J G O'Grady, M A Heneghan, K Agarwal, V Aluvihare, P Srinavasan, A Prachalias, N Heaton, A R Suddle. Institute of Liver Studies, King's College Hospital, London, UK

Introduction In selected patients, liver transplantation (LT) is considered a curative treatment for hepatocellular carcinoma (HCC). With the application of strict eligibility criteria the risk of tumour recurrence can be minimised but never entirely eliminated. Historically, HCC recurrence is associated with a poor prognosis; however the new and evolving systemic therapies may improve outcomes. Hence, there may be a potential beneficial role for early diagnosis. Although, serum alpha-fetoprotein (AFP) measurement is commonly employed in HCC surveillance in at-risk patients, its role following LT for HCC is not clearly defined. Therefore, we have explored (1) the utility of AFP as a biomarker for tumour recurrence following LT in patients with HCC and (2) the influence of liver disease aetiology on its diagnostic performance.

Methods The clinical characteristics, laboratory parameters and outcomes of 302 patients with HCC who underwent LT between January 1999 and July 2011 at our institution were reviewed. Serum AFP levels following LT were analysed and the area under a receiveroperator characteristic curve (AUC) calculated to assess the performance of AFP as a diagnostic test for HCC recurrence.

Results Recurrent HCC was observed in 13\% of patients during the follow-up period. Recurrence was associated with higher pre-LT AFP (98 ng/ml vs $11.5 \mathrm{ng} / \mathrm{ml}, \mathrm{p}>0.001)$, vascular invasion in the liver explant, and greater tumour size. Following LT, the median time from initial rise in serum AFP to the diagnosis of recurrence was 16 weeks. In our cohort, AFP was an effective predictor of HCC recurrence (AUC 0.843) and using a cut-off value of $\geq 10 \mathrm{ng} / \mathrm{ml}$ was an excellent exclusion test (negative predictive value 0.94). Furthermore, its performance was superior in patients with AFPsecreting tumours at LT, in comparison to those with non-secreting tumours (AUC 0.892 vs 0.710 ). Patients with chronic hepatitis C (CHC), in comparison to non-infected patients, were younger ( 56 years vs 60 years, $p=0.887$ ) and had a lower MELD score (13 vs $10 \mathrm{ng} / \mathrm{ml}, \mathrm{p}=0.002)$ but higher serum AFP (21 ng/ml vs $10 \mathrm{ng} / \mathrm{ml})$ at LT. However, there was no difference in recurrence rates between groups. In patients with $\mathrm{CHC}$, AFP was an inferior predictor of HCC recurrence, in comparison non-CHC patients (AUC 0.769 vs 0.887).

Conclusion We, therefore, conclude that serum AFP measurement is a potentially useful surveillance investigation for tumour recurrence post-LT for HCC particularly in patients without CHC.

Competing interests None declared.

\section{PTU-057 SPLIT LIVER TRANSPLANT RECIPIENTS ARE LESS LIKELY TO REQUIRE PERI-OPERATIVE RENAL REPLACEMENT THERAPY THAN FULL-SIZE LIVER TRANSPLANT CONTROLS}

doi:10.1136/gutjnl-2012-302514c.57

\footnotetext{
1,2 J A Leithead, ${ }^{* 1,2} \mathrm{M}$ J Armstrong, ${ }^{1,2} \mathrm{C}$ Corbett, ${ }^{1} \mathrm{M}$ Andrew, ${ }^{1} \mathrm{C}$ Kothari, ${ }^{1} \mathrm{~F}$ Tinti, ${ }^{2} \mathrm{~B}$ K Gunson, ${ }^{1} \mathrm{D}$ Mirza, ${ }^{1} \mathrm{P}$ Muiesan, ${ }^{1} \mathrm{~J}$ W Ferguson. 'Liver Unit, Queen Elizabeth Hospital, Birmingham, UK; ${ }^{2}$ NIHR Biomedical Research Unit and Centre for Research, University of Birmingham, Birmingham, UK
}

Introduction Several small studies have suggested that split liver transplant (SLT) recipients have an increased frequency of peri-operative acute kidney injury (AKI). However, given that hepatic ischaemia-reperfusion injury may play a role in the pathogenesis of peri-transplant AKI, the optimal donor selection of split liver transplantation could have a favourable impact on renal outcomes. Our aim was to compare renal outcomes in SLT recipients with matched full-size liver transplant (FSLT) controls.

Methods Single-centre study of 72 patients who received a SLT for chronic liver disease 01/2007-06/2011. 72 FSLT (Donation after Brain Death) controls were matched by propensity-risk-score. Definitions: AKI, peak creatinine $\geq 2$ times baseline; chronic kidney disease (CKD), MDRD4 eGFR $<60 \mathrm{ml} / \mathrm{min} / 1.73 \mathrm{~m}^{2}$.

Results SLT recipients and FSLT controls were well matched on confounders. Split liver grafts had a younger donor age $(p<0.001)$, longer cold ischaemic time $(p=0.026)$ but similar warm ischaemic time $(p=0.213)$. There was no difference in the intra-operative red cell concentrate requirements between the groups $(p=0.460)$. During the immediate post-operative period, the median peak AST was $1156 \mathrm{U} / \mathrm{L}$ for SLT recipients and $1124 \mathrm{U} / \mathrm{L}$ for FSLT controls $(p=0.960)$. The frequency of re-laparotomy for bleeding $(p=0.310)$, primary non-function $(p=1.000)$, sepsis $(p=0.643)$ and biliary complications were comparable $(p=1.000)$. Estimated 3-year patient survival was $90.0 \%$ and $91.8 \%$ for SLT recipients and controls, respectively (log-rank $\mathrm{p}=0.400$ ). Peri-operative and long-term renal outcomes are outlined in Abstract PTU-057 table 1. There was no difference between the two groups, with the exception of renal replacement therapy; SLT recipients were less likely to require peri-operative renal replacement therapy than FSLT controls $(p=0.048)$.

Abstract PTU-057 Table 1 Post transplant renal outcomes in SLT recipients and FSLT controls

\begin{tabular}{llll}
\hline & SLT & FSLT & p Value \\
\hline Peri-operative renal function & & & \\
$\quad$ Median peak creatinine $(\mu \mathrm{mo} / /)$ & 113 & 116 & 0.585 \\
$\quad$ Median change in creatinine from baseline (\%) & +27 & +43.6 & 0.653 \\
AKI (\%) & 29.2 & 34.7 & 0.475 \\
$\quad$ Renal replacement therapy (\%) & 11.1 & 23.6 & $\mathbf{0 . 0 4 8}$ \\
Long-term renal function & & & \\
$\quad$ Mean 1-month eGFR (ml/min/1.73 m²) & 83 & 81 & 0.773 \\
3-year cumulative incidence of CKD (\%) & 32.5 & 28.7 & 0.534 \\
\hline
\end{tabular}

Conclusion SLT recipients are less likely to require peri-operative renal replacement therapy than well matched FSLT recipients. Higher graft quality and/or smaller graft volume may have a beneficial renal-sparing effect.

Competing interests None declared.

\section{PTU-058 COMMUNITY BASED RANDOM BLOOD ALCOHOL LEVEL TESTING WITHOUT PRIOR NOTICE DETECTS CONCEALED ALCOHOL INGESTION IN PATIENTS WITH ALCOHOLIC LIVER DISEASE AWAITING LIVER TRANSPLANTATION}

doi:10.1136/gutjnl-2012-302514c.58

\author{
J P Bate, ${ }^{*}$ K Rothwell, M A Aldersley. Liver Unit, St James's University Hospital \\ Leeds, UK
}

Introduction Alcoholic liver disease (ALD) is a common indication for liver transplantation (LT) but recidivism is a significant concern There is a need for careful assessment and support prior to LT, with monitoring for ongoing alcohol use an important aspect of care for patients on the waiting list (WL).

Methods Patients listed for LT in the UK are required to sign a contract committing them to lifelong abstinence from alcohol 